\title{
Testing the models of CV evolution
}

\author{
A. F. Pala*1, A. Ederoclite ${ }^{2}$, N. P. Gentile Fusillo ${ }^{1}$, H. Vázquez Ramió ${ }^{2}$, R. Raddi ${ }^{1}$, \\ J. Abril' ${ }^{2}$, B. T. Gänsicke ${ }^{1}$, A. Rebassa-Mansergas ${ }^{3}$ \\ ${ }^{1}$ Department of Physics, University of Warwick, Coventry, CV4 7AL, UK \\ ${ }^{2}$ Centro de Estudios de FïÄśsica del Cosmos de Aragóon, Plaza San Juan 1, Planta-3, E-44001 \\ Teruel, Spain \\ ${ }^{3}$ Departament de Física, Universitat Politécnica de Catalunya, c/Esteve Terrades 5, E-08860 \\ Castelldefels, Spain \\ E-mail: A.F.Pala@warwick.ac.uk
}

\begin{abstract}
The study of Cataclysmic Variables (CVs) is crucial to test our understanding of binary evolution and its application to many astrophysical phenomena, such as short gamma-ray bursts, X-ray transients and, more important, Supernovae Ia, our yardsticks for measuring distances. Yet, the predicted major component of the present-day CV population, the so-called "period bouncers" (CVs containing a white dwarf and a degenerate donor), has not been detected, highlighting a major discrepancy between theory and observations. We present here $\mathrm{CHiCaS}$, the Compact binary HIgh CAdence Survey, which will perform three hours of uninterrupted time series photometry over 136 square degrees of the sky with JAST/T80Cam. By the end of next year, this program will deliver one minute cadence light curves for $\simeq 2.5$ million objects as faint as $g \simeq 21.5$, along with full colour information. Via detection of their eclipses, CHiCaS will finally, and unambiguously identify the predicted large population of period bouncers. The identification of the missing population will provide an observational support for the current models for the mechanisms of angular momentum loss in compact binaries, which also describe the evolution of all kind of binaries. CHiCaS will also offer a complete and unbiased view into the short term variability of thousands of binaries, eclipsing systems, pulsating stars and CVs in the period gap, which will allow to improve our knowledge of these objects and to carry out additional tests on CV evolution.
\end{abstract}

The Golden Age of Cataclysmic Variables and Related Objects IV

11-16 September, 2017

Palermo, Italy

* Speaker. 


\section{Introduction}

Cataclysmic variables (CVs) are short-period ( $\simeq 80 \mathrm{~min}$ to $\simeq$ day) interacting binaries contain a white dwarf accreting from a low-mass companion star. Since both stellar components are structurally simple and well understood and there is a large number of relatively bright CVs known, it is possible to carry out detailed observational population studies. Therefore CVs are one of the best-suited classes to test, constrain, and further develop our understanding of the evolution of all types of compact binaries, such as black hole binaries, X-ray transients, double degenerates and, more importantly, the pathways towards Type Ia Supernova (SN Ia), our yardsticks for measuring distances on cosmological distance scales.

Over the last decades, improved technologies and the advent of large spectroscopic and timedomain surveys have highlighted major discrepancies between the standard model of CV evolution and the observed CV population, with important implications for many astrophysical fields. In other words, the failure of the theory describing such simple systems undermines our faith in understanding the evolution of more complex binaries, particularly SNe Ia, the fundamental tool to probe the physical properties of the Universe. Against this background, it is both timely and important to thoroughly investigate the disagreements between the observations and the theory describing compact binary evolution.

The evolution of CVs (and all other compact binaries) is dictated by angular momentum loss (AML) which results in continuous mass loss from the donor star, sets the mass accretion rate $(\dot{M})$, and results in a decrease of the orbital period $\left(P_{\text {orb }}\right)$. The evolution proceeds in this way until the system reaches the "period minimum" at $\simeq 70-75$ min (Knigge et al., 2011; Goliasch \& Nelson, 2015; Kalomeni et al., 2016). At this stage, the secondary mass has become so low ( $M_{\mathrm{sec}} \simeq$ $0.06 \mathrm{M}_{\odot}$ ) that core $\mathrm{H}$-burning ceases, and the star turns into a brown dwarf, i.e. a degenerate object, i.e. expands in response to the mass loss. Consequently, the orbital separation and period increase as angular momentum loss continues, driven by gravitational wave radiation. These objects are called "period bouncers" and are expected to make up for $\simeq 40-70 \%$ (Kolb, 1993; Knigge et al., 2011; Goliasch \& Nelson, 2015) of the present Galactic CV population. However, despite more than 40 years of extensive research on CVs and more than 1400 systems with a measured orbital period (Ritter \& Kolb, 2003), only a handful of period bouncer candidates have been identified so far (e.g. Patterson et al. 2005; Unda-Sanzana et al. 2008; Littlefair et al. 2006; Patterson 2011; Kato et al. 2015, 2016; McAllister et al. 2017; Neustroev et al. 2017, and many others), therefore enlarging the sample of post-bounce CVs is important in order to validate a fundamental prediction of the current models of compact binary evolution.

The lack of period bouncers in the observed CV population could be explained by limitations in the detection methods followed so far. In fact, CVs are typically identified based on their accretion luminosity, thus favouring the discovery of long-period systems, that are characterised by mass accretion rates of about one order of magnitude higher than those of short-period CVs (Townsley \& Gänsicke, 2009; Pala et al., 2017). The identification of period bouncers is therefore challenging since they are expected to be intrinsically faint $(V \simeq 18.5-20.5 \mathrm{mag}$, Pala et al. 2017). $\mathrm{CVs}$ are also identified thanks to their disc outbursts, i.e. thermal instabilities in the disc which cause a variation in the mass transfer rate through it (Osaki, 1974; Hameury et al., 1998; Meyer \& Meyer-Hofmeister, 1984) during which the system typically brightens by $2-5$ magnitudes, oc- 
casionally up to 9 magnitudes (Warner, 1995; Maza \& Gonzalez, 1983; Templeton, 2007). This identification method disfavours the discovery of low mass transfer rate systems, such as period bouncers, which are expected to rarely, maybe never, experience outbursts.

Another possibility is that our understanding of binary evolution is incomplete and the predicted period bouncers actually do no make up for the majority of the present day CV population. Critically uncertain areas in the theoretical modelling are (i) the evolution of stars undergoing mass loss, (ii) the common envelope phase during which the progenitor evolves up the giant branch and engulfs the secondary star, and (iii) the mechanisms of angular momentum loss. The number of identified period bouncers is still far from the fraction predicted by theory $(\simeq 40-70 \%$, Kolb 1993; Knigge et al. 2011; Goliasch \& Nelson 2015) and the lack of these highly evolved CVs undermines our confidence in the current models of compact binary evolution and formation, which are also used to describe the evolution of more complex systems, such as black hole binaries, X-ray transients, milli-second pulsars or SN Ia progenitors. Similarly, neutron star and black hole mergers are the sources of gravitational wave emission, and the recent detections of the first gravitational wave sources by the LIGO and Virgo experiments (Abbott et al., 2016a,b, 2017; The LIGO Scientific Collaboration et al., 2017; The LIGO Scientific Collaboration \& The Virgo Collaboration, 2017) underlines the need for a proper understanding of binary formation and evolution.

Therefore the identification of period bouncer CVs is important in order to validate a fundamental prediction of the current models of compact binary evolution and we here present the Compact binary HIgh CAdence Survey (CHiCaS), a new observing program aimed to unambiguously identify, for the first time, the elusive population of period bouncers.

\section{The search for period bouncers}

Period bouncers have orbital periods in the range $80 \mathrm{~min} \lesssim P_{\text {orb }} \lesssim 120 \mathrm{~min}$, and are characterised by low mass accretion rates $\left(\dot{M} \simeq 10^{-11} \mathrm{M}_{\odot} \mathrm{yr}^{-1}\right)$ which makes them undetectable through the usual methods used for identify CVs. In fact, their accretion discs and secondary stars are expected to be very faint, and the systems would hence resemble cool, faint and apparently single white dwarfs. However, CVs are tight binaries with orbital separations of the order of the solar radius, and $\simeq 15-20$ per cent of them will be seen under the right inclination from the Earth, in such a way that the Earth-sized white dwarfs will be eclipsed for a few minutes by their larger Jupitersized brown dwarf donors every $\simeq 90 \mathrm{~min}$. This is very important because from the eclipse light curve analysis it is possible to determine the orbital period and the masses of both the white dwarf and the donor star (e.g. Littlefair et al. 2006), where the donor mass is critical to unambiguously distinguish between period bouncers from $\mathrm{CV}$ s that have not evolved through the period minimum yet (see for example figure 5 from Howell et al. 2001).

Therefore, the ingredients to successfully identify this elusive major component of the CV population are deep observations, able to observe objects as faint as $V \simeq 20.5 \mathrm{mag}$ (as expected for period bouncer CVs, Pala et al. 2017), in combination with high-time resolution photometry, in order to detect the eclipses of period bouncer CVs lasting a few minutes. Furthermore, CVs are relatively rare objects, characterised by a low space density $\left(\rho \simeq 2 \times 10^{-4} \mathrm{pc}^{-3}\right.$, de Kool 1992) and hence large area of the sky needs to be surveyed to build a significant statistical sample, necessary to perform a stringent test of the predictions from population synthesis models. The survey area 
Table 1: Properties of the JAST/T80Cam.

\begin{tabular}{lc}
\hline FoV & $1.4^{\circ} \times 1.4^{\circ}$ \\
CCD format & $9216 \mathrm{pix} \times 9232 \mathrm{pix}$ \\
Pixel size & $10 \mu \mathrm{m} \times 10 \mu \mathrm{m}$ \\
Pixel scale & $0.55^{\prime \prime} /$ pixel \\
Number or read modes & 16 \\
Redout time (\# 5) & $12 \mathrm{~s}$ \\
Readout noise (\# 5) & $3.4 e^{-}(\mathrm{RMS})$ \\
Minimum exposure time & $0.1 \mathrm{~s}$ \\
\hline
\end{tabular}

Table 2: Effective wavelengths and passbands for the JAST/T80Cam filters employed in CHiCaS.

\begin{tabular}{lcc}
\hline Filter & $\begin{array}{c}\text { Effective wavelength } \\
(\AA)\end{array}$ & $\begin{array}{c}\text { Band width } \\
(\AA)\end{array}$ \\
\hline$u J A V A$ & 3485 & 508 \\
$g S D S S$ & 4803 & 1409 \\
$r S D S S$ & 6254 & 1388 \\
$J 0660$ & 6600 & 145 \\
$i S D S S$ & 7668 & 1535 \\
$z S D S S$ & 9114 & 1409 \\
\hline
\end{tabular}

should be observed at least for three hours, in order to maximise the probability to detect at least one eclipse per system from which the orbital period is then unambiguously determined. Finally, multi-band photometry is helpful to select period bouncers candidates from their colours.

On January 2017, we have started CHiCas, a high-cadence photometric survey carried out at the Javalambre Observatory using T80Cam at the Javalambre Auxiliary Survey Telescope (JAST).

JAST is a $80 \mathrm{~cm}$ telescope located at the newly born Observatorio Astrofísico de Javalambre (Teruel, Spain, Cenarro et al. 2010), managed and constructed by the Centro de Estudios de Física del Cosmos de Aragón (CEFCA). JAST has a large FoV of $1.4^{\circ}$ in diameter and its equipped with T80Cam, a large-format $9.2 \mathrm{k} \times 9.2 \mathrm{k}$ pixel CCD camera that, at the time of its purchase (2011), was the largest CCD detector employed in astronomy.

The T80Cam CCD camera (Table 1) has pixels of $10 \mu \mathrm{m}$ size and it is read simultaneously from 16 ports which allow different readout times, the shortest of which is $3 \mathrm{~s}$, with typical readout noise of $\simeq 4 e^{-}$(RMS). The observations carried out in this work used the readout mode \# 5, i.e. with $12 \mathrm{~s}$ readout time and $\simeq 3.4 e^{-}$(RMS) readout noise. Moreover, T80Cam is equipped with a set of 12 filters, some of them overlapping with the SDSS filters. Thanks to this characteristic and its large FoV, T80Cam is the ideal instrument to perform high-time resolution photometry on large areas of the sky and to acquire the multi-band photometric observations required to identify period bouncers.

By the end of 2018, CHiCaS will perform high-time resolution photometry at one minute cadence of 68 fields, corresponding to a total of 136 square degrees. Reaching a limiting magnitude 
of $g \simeq 22 \mathrm{mag}$ in dark time and $g \simeq 21.5 \mathrm{mag}$ in gray time, the program will deliver light curve for $\simeq 2.5$ million objects. Moreover, using the Sloan ugriz and the $\mathrm{H} \alpha$ filters (Table 2), full colour information will be provided for every object in the survey footprint.

The fields have been identified within low galactic latitude $\left(|b| \simeq 15^{\circ}\right)$ regions and, using the three-dimensional map of interstellar dust reddening based on Pan-STARRS 1 and 2MASS photometry (Green et al., 2015), low reddening $(E(B-V)<0.05)$ fields have been selected. Each field has been inspected by eye using DSS (Digitized Sky Survey) images to ensure that it contains a fair amount of objects and, at the same time, it is not too crowded and allows to perform accurate photometry.

The observing strategy consists in first acquiring one image in each of the following filters: the blue filter ( $u J A V A$ ), all the Sloan filters ( $g S D S S$, rSDSS, iSDSS and zSDSS) and the H $\alpha$ filter (J0660), useful to identify $\mathrm{H} \alpha$ emission from the disc, one of the most prominent feature in a CV spectrum. From this multiband photometry, following the method from Gentile Fusillo et al. (2015), we identify white dwarfs and CVs in our fields. Using the gSDSS filter, we then perform uninterrupted time-series photometry of each FoV for 3 hours, thus maximising the probability to identify at least one eclipse per period bouncer.

Given the theoretical CVs space density and assuming to detect systems out to $\simeq 350 \mathrm{pc}$, we expect to find $0.48 \mathrm{CVs}$ per square degree, that is a total of $67 \mathrm{CVs}$. Assuming that period bouncers make up for 70 per cent of the observed CVs and taking into account the probability that the system must be seen under the right inclination $(\simeq 16 \%)$ to detect eclipses, we expect to identify eight period bouncers at the end of the program.

\subsection{Scientific objectives}

The main goal of this program is to carry out a critical test of binary evolution models by searching for the missing CV population of period bouncers. Moreover, at the same time, $\mathrm{CHiCaS}$ will deliver a large amount of data which will provide a valuable legacy of additional scientific results:

- Eclipsing systems are the gold standard to measure precise stellar masses and radii (Torres et al., 2010). Our survey will detect thousands of eclipsing binaries, including detached, semi-detached and contact systems. Of particular interest are double white dwarf binaries, which will provide the opportunity to investigate one of the possible channels leading to SN Ia explosions.

- Asteroseismology is a powerful tool to study stellar interiors and determine physical parameters, including masses, temperatures, and magnetic fields. From our program we will obtain light curves of different kind of pulsators, including $\delta$ Scuti and GW Virginis stars. Furthermore, using the method from Gentile Fusillo et al. (2016), we can also identify ZZCeti stars, investigating the fundamental properties (internal structure, core and envelope mass and magnetic field) of these pulsating white dwarfs.

- The period gap is one of the most prominent feature in the CV orbital period distribution. In this period range, CVs evolve as detached white dwarf plus main sequence star systems, 
Table 3: Summary of the observations of CHiCaS0650.

\begin{tabular}{llll}
\hline Date & Filter & $N_{\text {Exp }}$ & $t_{\operatorname{Exp}}[\mathrm{s}]$ \\
\hline $2017-02-14$ & $u J A V A$ & 3 & 300 \\
$2017-02-14$ & $g S D S S$ & 3 & 60 \\
$2017-02-14$ & $r S D S S$ & 3 & 57 \\
$2017-02-14$ & $i S D S S$ & 3 & 54 \\
$2017-02-14$ & $z S D S S$ & 3 & 363 \\
$2017-02-14$ & $J 0660$ & 3 & 366 \\
\hline $2017-02-24$ & $g S D S S$ & 180 & 60 \\
\hline
\end{tabular}

hosting secondaries of spectral type M4-M6 (Zorotovic et al., 2016). Thanks to the multiband images we acquire in our survey, we can easily identify these important objects from their colour (Rebassa-Mansergas et al., 2012). Their orbital periods will be determined from the ellipsoidal modulation in their light curves and, with only one follow-up spectrum, we can determine the spectral type of the secondary. In this way, we will identify the CVs that are presently crossing the period gap, carrying out another powerful test of $\mathrm{CV}$ evolution theory.

\subsection{Observations}

During the first ten months of the survey, multi-band and time-series photometry have been acquired for 33 and 14 fields, respectively. In this Section, we present the results of the observations of the field CHiCaS J065048+230614 (hereafter CHiCaS0650), for which both multiband and time-series photometry are available.

Multi-band photometry of CHiCaS0650 has been acquired on February 14, 2017, through thin clouds. Time-series photometry has been obtained on February 24, 2017. Seeing was about $3^{\prime \prime}$ and sky was again covered by thin clouds. The summary of the observations is reported in Table 3.

The basic data reduction is performed by CEFCA. This includes bias removal, flat fielding and the astrometric solution. Moreover, the sets of three exposures per filter, acquired to construct the colour-colour diagrams, are combined into one image for each filter with higher SNR (Figure 1). The reduced data can be retrieved from their database.

The photometry and light curve extraction was carried out following the subsequent procedure:

- a first run of SEXTRACTOR (Bertin \& Arnouts, 1996) was performed on each image assuming a photometric aperture of five pixels in order to generate a preliminary catalogue containing the position and the full width half maximum (FWHM) of each source. In order to maximised the signal-to-noise ratio (SNR), the median value of all the measured FWHMs was then multiplied by 1.5 and was used as input aperture for a second run of SEXTRACTOR. This returned a catalogue containing the positions and the instrumental magnitudes of all the identified sources in each image.

- The catalogue of each image generated in the previous step was then cross-matched with the APASS (AAVSO Photometric All-Sky Survey, Henden \& Munari 2014) catalogue, which 


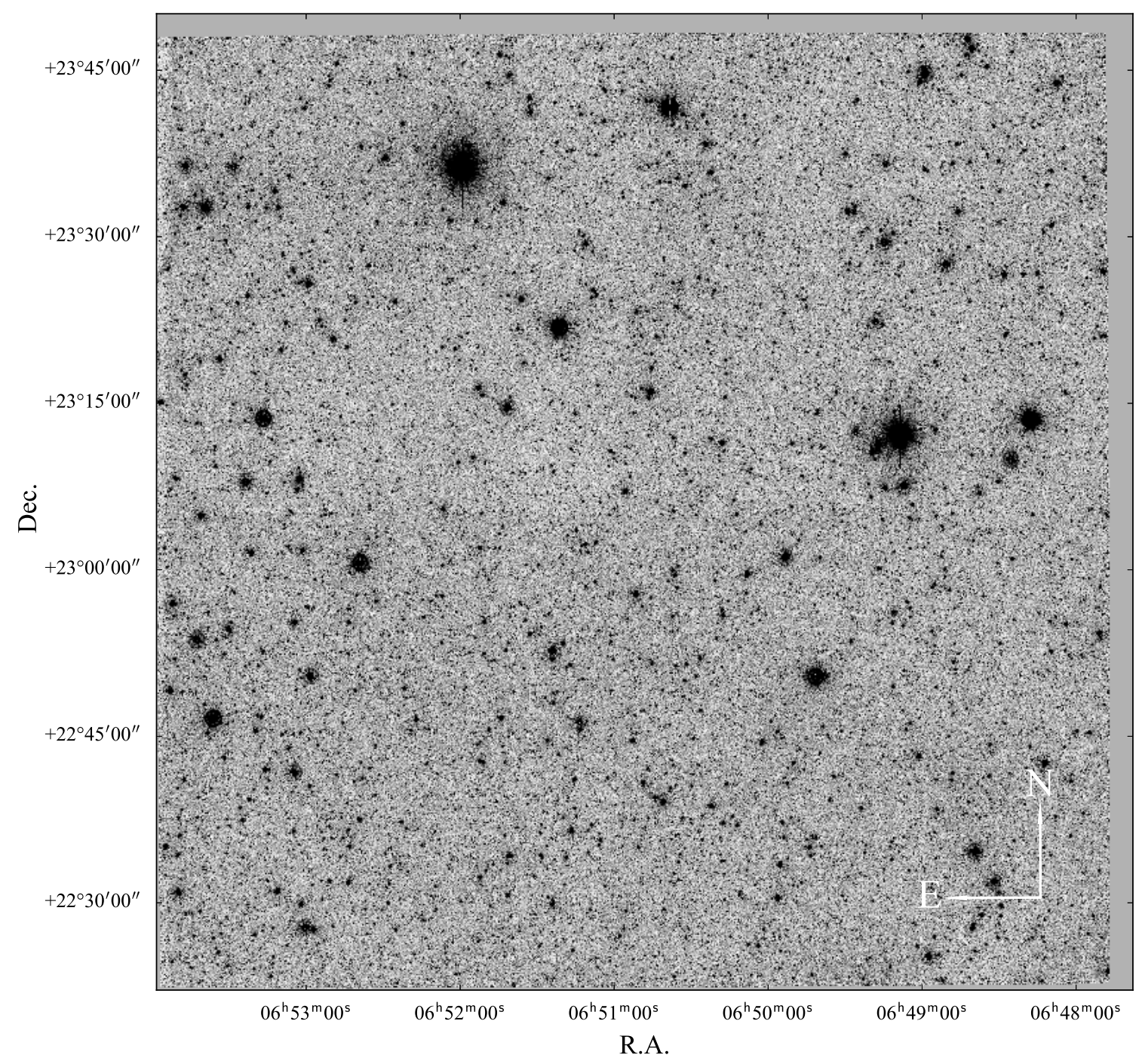

Figure 1: The gSDSS-band image of CHiCaSJ0650, obtained from the combination of three exposures of $60 \mathrm{~s}$ each. The field of view is $1.4^{\circ} \times 1.4^{\circ}$ wide and contains $\simeq 38000$ sources as faint as $g \simeq 21.5 \mathrm{mag}$.

provides photometry in the $g, r$ and $i$ filters. In the case of CHiCaS0650, 4160 reference stars were identified. For each one of them, the difference between the instrumental magnitude and the tabulated value was calculated. The median value was then added to the instrumental magnitude of all detected sources to perform the photometric calibration in the corresponding filter band.

- The previous steps returned the photometric calibration for both the multi-band and the time-series observations. The light curve extraction was then performed using the gSDSSband image of CHiCaSJ0650 acquired on 2017-02-14 as a reference. Each detected source in this image was crossmatched with the catalogue of the time-series frames, thus providing the corresponding light curve. 

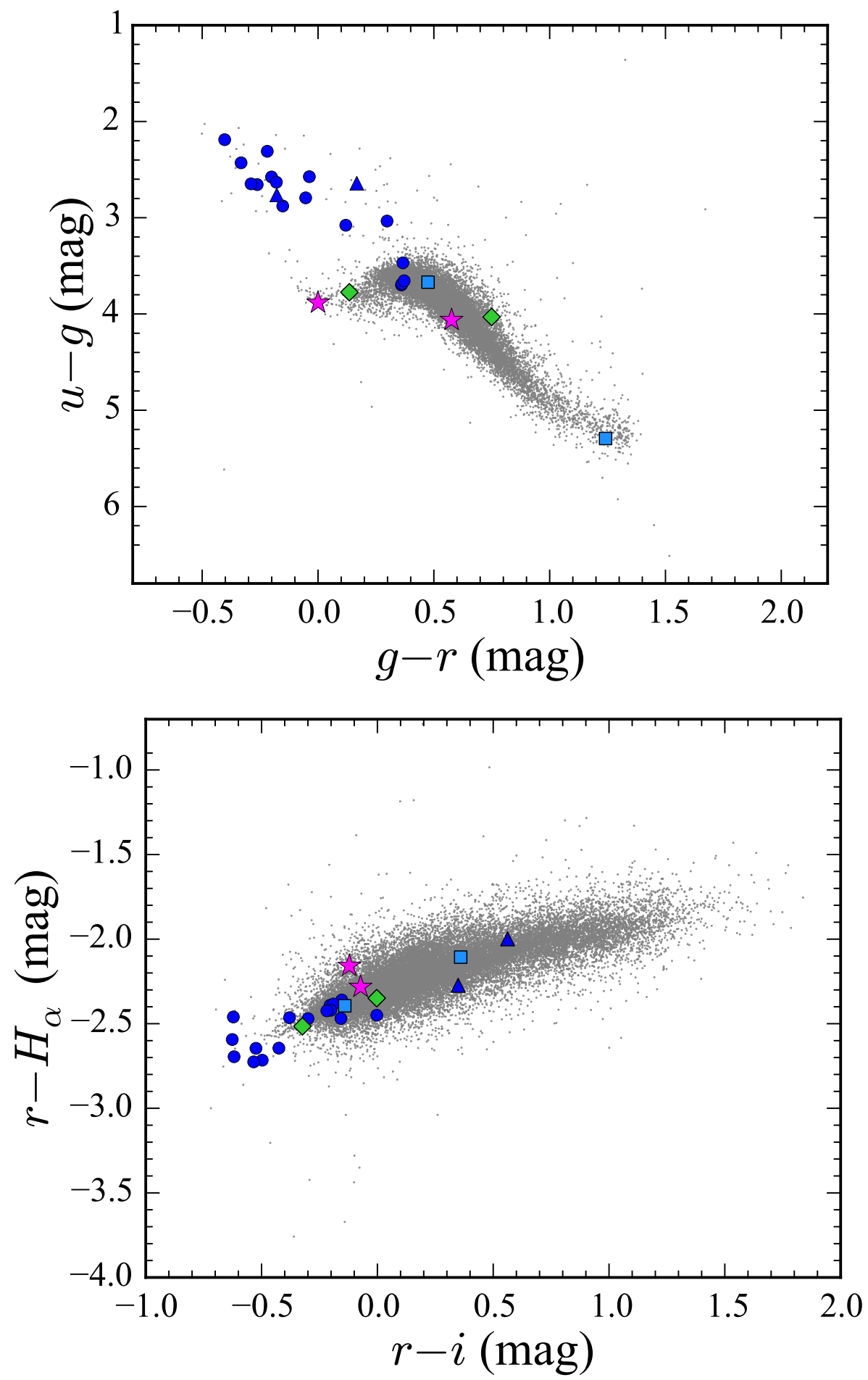

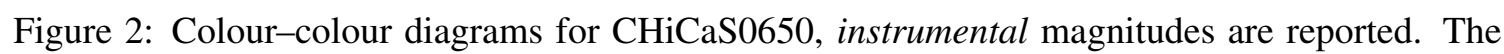
blue circles are the white dwarf candidates while the blue triangles are the two CV candidates. The stars, the squares and the diamonds are, respectively, the contact binaires and the flaring and pulsating stars whose light curves are shown in Figure 5. 
Table 4: White dwarf candidates from CHiCaS0650.

\begin{tabular}{cccc}
\hline$\alpha$ & $\delta$ & $g(\mathrm{mag})$ & $P_{\mathrm{WD}}(\%)$ \\
\hline $06: 47: 48.17$ & $23: 14: 08.36$ & 18.73 & 88.1 \\
$06: 47: 53.13$ & $22: 57: 57.83$ & 19.37 & 87.3 \\
$06: 48: 33.62$ & $23: 25: 43.66$ & 20.20 & 89.8 \\
$06: 48: 46.22$ & $23: 03: 03.62$ & 17.45 & 95.9 \\
$06: 49: 28.66$ & $23: 38: 36.47$ & 15.78 & 87.5 \\
$06: 49: 32.81$ & $23: 35: 50.83$ & 17.65 & $90.0^{*}$ \\
$06: 49: 49.51$ & $23: 27: 42.29$ & 20.09 & 84.5 \\
$06: 50: 09.61$ & $23: 23: 50.94$ & 20.33 & $94.6^{*}$ \\
$06: 50: 21.42$ & $23: 45: 09.28$ & 19.88 & 93.1 \\
$06: 50: 25.20$ & $23: 10: 51.06$ & 19.04 & 99.3 \\
$06: 50: 41.30$ & $23: 09: 54.10$ & 18.58 & 99.9 \\
$06: 50: 44.73$ & $23: 00: 19.64$ & 20.31 & 83.4 \\
$06: 51: 22.27$ & $22: 26: 09.05$ & 16.39 & 74.4 \\
$06: 51: 45.10$ & $23: 36: 48.57$ & 19.76 & 93.1 \\
$06: 52: 03.05$ & $22: 38: 52.09$ & 20.36 & 93.9 \\
$06: 52: 04.66$ & $23: 43: 55.11$ & 19.73 & 97.6 \\
$06: 52: 23.38$ & $23: 00: 27.62$ & 20.21 & 77.1 \\
$06: 53: 28.51$ & $23: 13: 29.79$ & 20.20 & 97.5 \\
\hline
\end{tabular}

Notes. The systems highlighted with a star are the two CV candidates.

\subsection{Preliminary results}

\subsubsection{Colour-Colour Diagrams}

Figure 2 shows the colour-colour diagrams for the $\simeq 38000$ sources we detected in CHiCaS0650. We here report the instrumental magnitudes since a photometric calibration has not yet been performed for the $U J A V A$ and $J 0660$ bands. Nonetheless, this does not affect the following analysis since it would only result in a shift of the $y$-axis values by a fixed amount, without changing the shape of the distribution.

White dwarf candidates can be selected thanks to their colours since they occupy the bluer region (top left corner) of the $(g-r, u-g)$ space. However, additional constraints are required in order to discriminate these candidates from other blue objects that populate the same colour region, such as quasars. Since white dwarfs are nearby objects, they are characterised by high proper motions that can be used to distinguish them from these contaminants.

Gentile Fusillo et al. (2015) have developed a method that, by combining multi-band photometry and proper motions, provides the probability for a object to be a white dwarf $\left(P_{\mathrm{WD}}\right)$. Therefore, we retrieved the proper motion for the sources in CHiCaS0650 from the PPMXL catalogue (Roeser et al., 2010) and, following the method of Gentile Fusillo et al. (2015), we identified 18 stars with a probability of being a white dwarf $P_{\mathrm{WD}}>70 \%$.

These candidates are listed in Table 4. In the $(g-r, u-g)$ plane (top panel of Figure 2), 


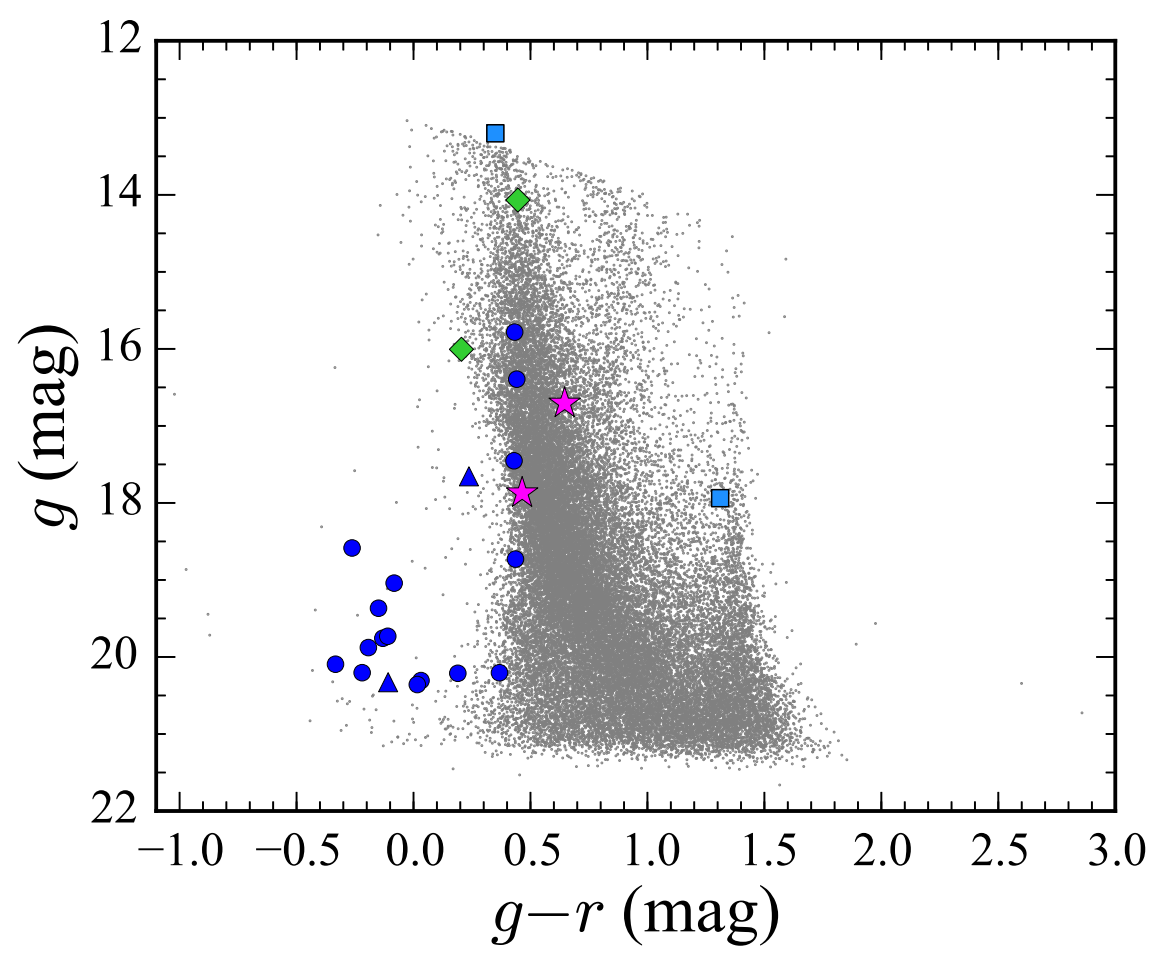

Figure 3: Colour-magnitude diagram for $\mathrm{CHiCaS}$, calibrated magnitudes are reported. The blue circles are the white dwarf candidates while the blue triangles are the two CV candidates. The stars, the squares and the diamonds are, respectively, the contact binaries and the flaring and pulsating stars whose light curves are shown in Figure 5.

the majority of the objects sit on the main sequence track while the white dwarf candidates (blue) occupy the bluer region. Two of them (triangles) are redder than the others, as suggested by their location on the right side of the $(r-i, r-H \alpha)$ plane, indicating the presence of an infrared excess. These systems are CVs candidates in which the excess could arise from a late-type companion. Alternatively, a white dwarf with a debris disc could also explain the observed colour excess (e.g. Girven et al. 2011). Since these two systems do no show eclipses (see below) that would confirm the presence of a companion, follow-up spectroscopy is required in order to establish the origin of the infrared excess.

Figure 3 shows the location of the white dwarf (blue dots) and the CV (blue triangles) candidates in the colour-magnitude diagram, for which calibrated magnitudes are reported. The diagram also shows that the limiting magnitude we could achieve on the night of the observation is $g \simeq 21.5 \mathrm{mag}$. 

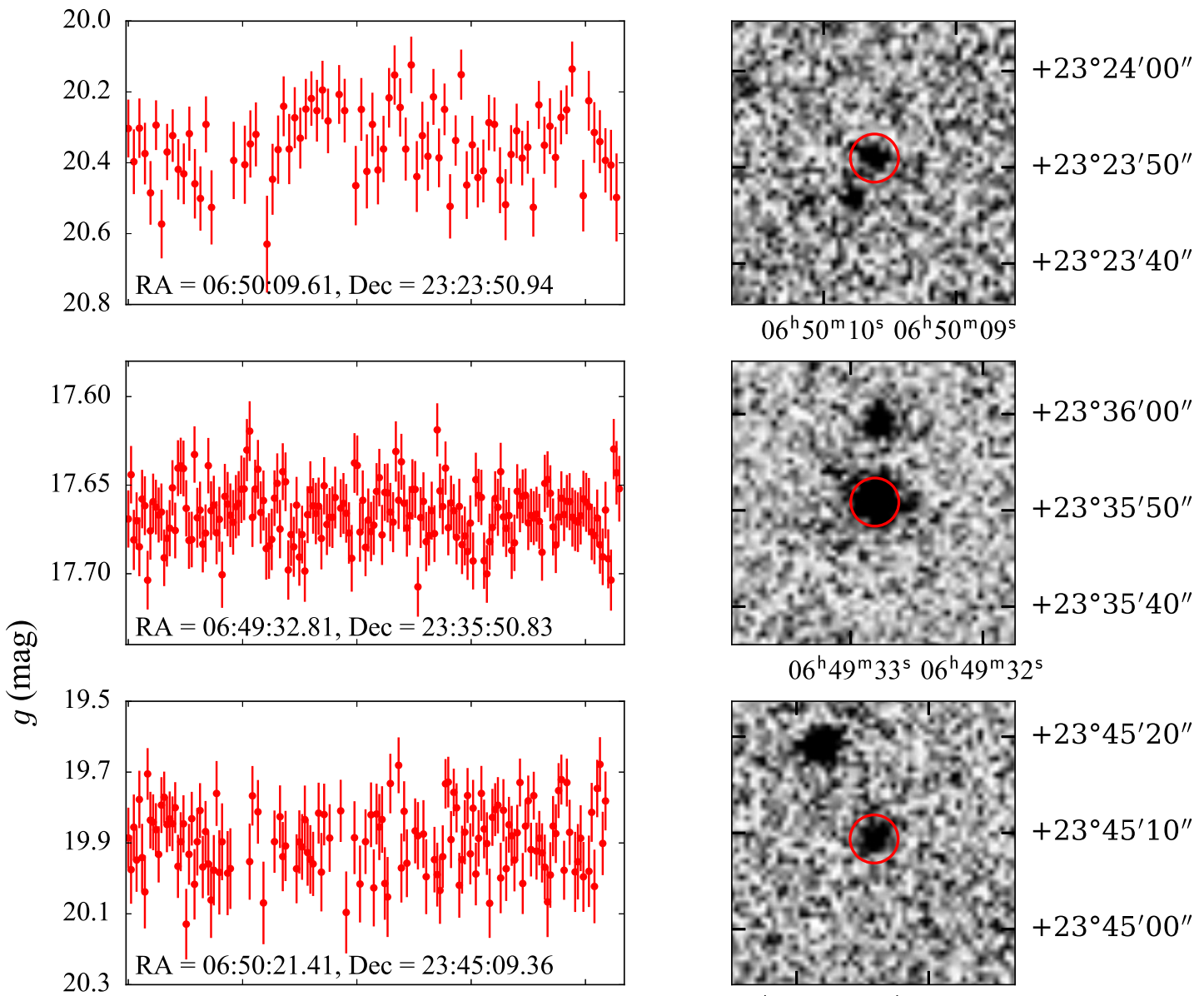

$+23^{\circ} 45^{\prime} 20^{\prime \prime}$
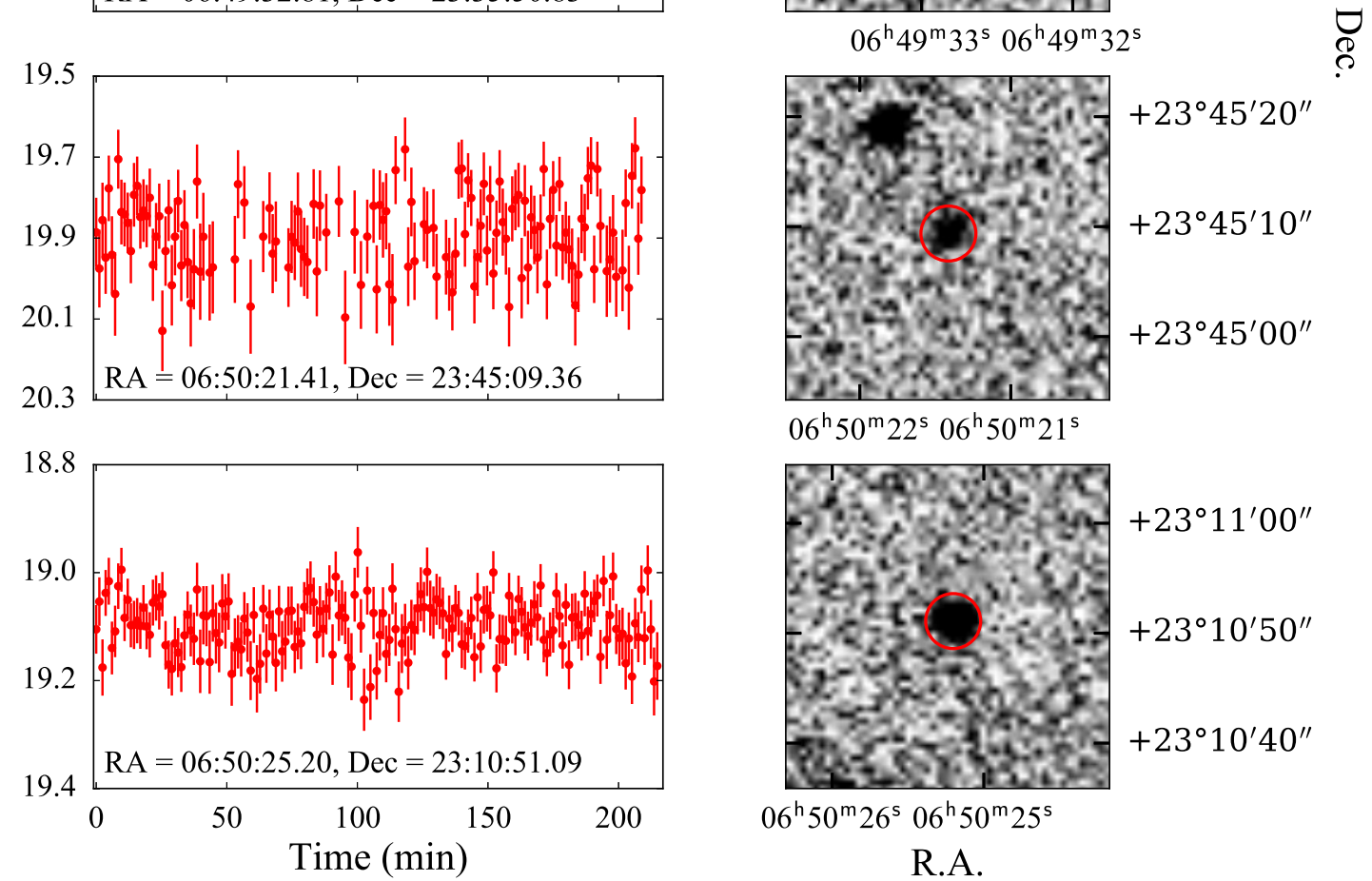

$06^{\mathrm{h}} 50^{\mathrm{m}} 22^{\mathrm{s}} 06^{\mathrm{h}} 50^{\mathrm{m}} 21^{\mathrm{s}}$

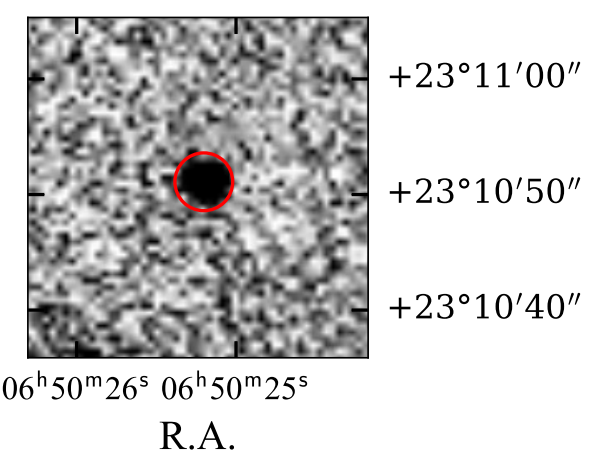

Figure 4: On the left, the light curves of the two CV candidates (first and second panels from the top) and sample light curves for two white dwarf candidates (third and fourth panels from the top) from CHiCaS0650 are plotted. On the right, for each star, a 30" $\times 30^{\prime \prime}$ cut-out from the gSDSSband image of CHiCaS0650 from 2017-02-14 is shown. All targets (red circles) are well resolved and are not blended with background sources. 

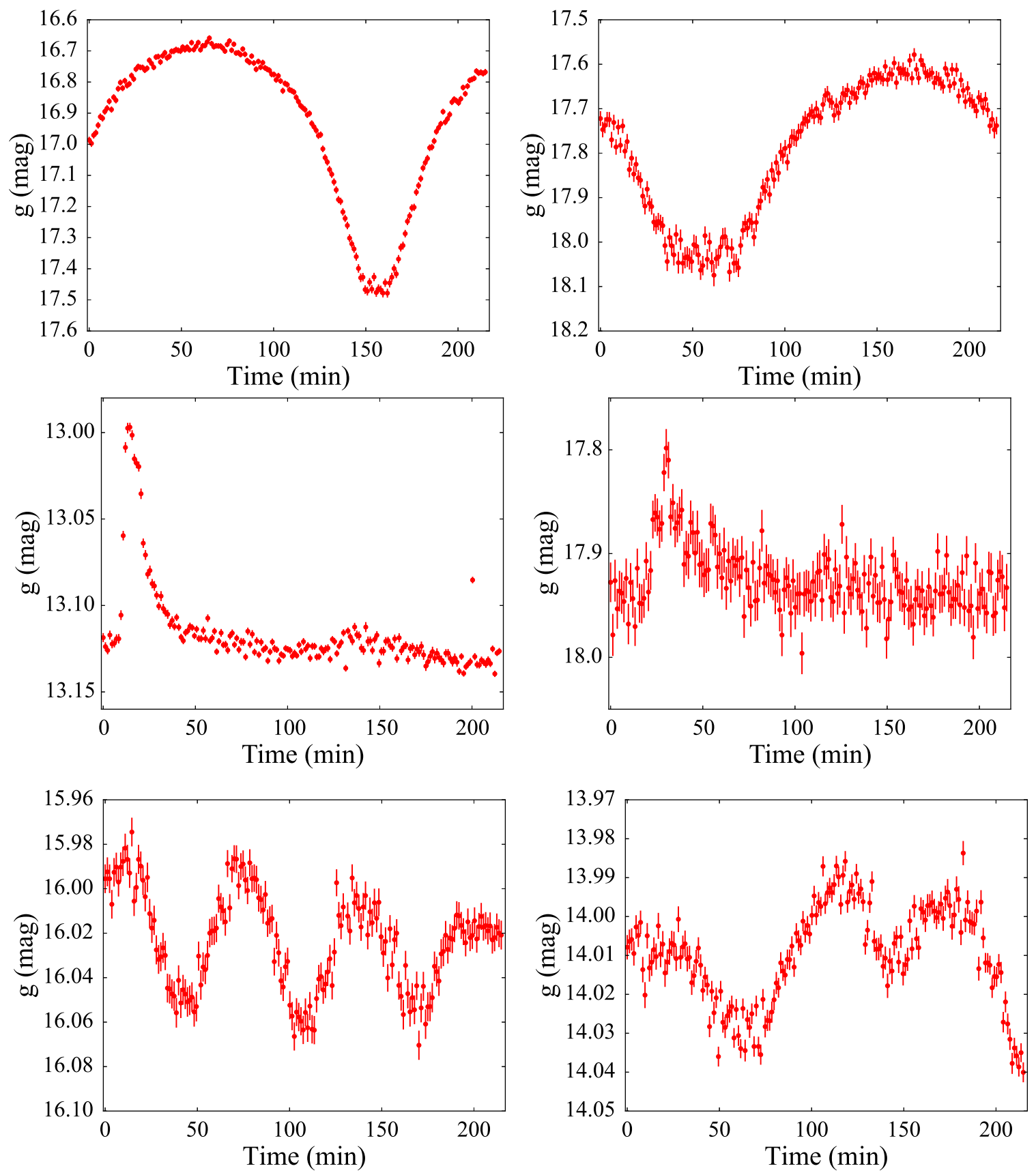

Figure 5: Sample light curves for six stars from CHiCaS0650 showing ellipsoidal modulations (top), flares (middle) and pulsations (bottom). 


\subsubsection{Time Series}

We show sample light curves of the objects with a probability of being a white dwarf $P_{\mathrm{WD}}>$ $70 \%$ in Figure 4 (top CV candidates, bottom white dwarf candidates). These objects are not listed either in Simbad or in the Montreal White Dwarf Database (Dufour et al., 2017) thus making this the first identification of these systems.

Moreover, by delivering light curves for all the objects in the field of view, our program provides a valuable legacy of ancillary data: we detected several contact binaries (Figure 5, top), flaring (Figure 5, middle) and pulsating stars (Figure 5, bottom). The analysis of these flares, pulsations and ellipsoidal modulations yields important additional scientific results, providing a large sample of stars for which magnetic fields, effective temperatures and masses can be accurately determined.

\section{Summary and future work}

$\mathrm{CHiCaS}$ is the the first systematic attempt to unambiguously identify the population of period bouncers. This survey will provide a complete and unbiased view into short term variability: by observing 136 square degrees of the sky at one minute cadence, it will deliver light curves and full colour information for $\simeq 2.5$ million stars as faint as $g \simeq 21.5$ mag.

From the analysis of the photometry for one of the 68 fields in the CHiCaS footprint, we have extracted the light curves for $\simeq 38000$ stars. The first observations of this program have shown that high-cadence time-series photometry with JAST/T80Cam allows the identification of faint CVs and white dwarfs, binary systems (either detached, semi/detached or in contact) and pulsating stars. Specifically, we have identified 16 new white dwarf candidates and two new CV candidates. Although no eclipse has been identified in the light curves of the two CV candidates, the two stars show an infrared excess which could imply the presence of either an companion or a debris disc. Follow-up observations are necessary to establish the origin of this colour excess.

Period bouncers are expected to be intrinsically faint and our results for systems of similar magnitudes have proved our capability to obtain the high-quality time-resolved data necessary to identify their possible eclipses. If period bouncers do represent a major component of the present day Galactic CV population, thanks to this photometric survey we will be able to identify them thus carrying out a critical test on the current models of $\mathrm{CV}$ evolution.

At present, the photometric calibration of the gJAVA, rJAVA and iJAVA bands has been carried out using APASS. Calibration of the UJAVA and $z J A V A$ bands requires SDSS photometry and several of our fields overlap with the SDSS footprint. Once multi-band photometry of these fields is acquired, we will be able to accurately calibrate all the bands. From this accurate calibration, using the method of Gentile Fusillo et al. (2016), we will be able to identify ZZ Ceti stars and to investigate the fundamental properties (internal structure, core and envelope mass and magnetic field) of these pulsating WDs.

Finally, one of the most prominent features in the CV orbital period distribution is the lack of systems in the period gap, where CVs evolve as detached white dwarf plus main sequence star binaries, hosting secondaries of spectral type M4-M6. Once also the $u$-band is calibrated, we will be able to identify these systems from their colours (Rebassa-Mansergas et al., 2012). Their orbital periods will be determined from the ellipsoidal modulation in their light curves and, with only one follow-up spectrum, we can determine the spectral type of the secondary. In this way, we will 
identify the CVs that are presently crossing the period gap, carrying out another stringent test on $\mathrm{CV}$ evolution.

\section{Acknowledgements}

The research leading to these results has received funding from the European Research Council under the European Union's Seventh Framework Programme (FP/2007-2013) / ERC Grant Agreement n. 320964 (WDTracer).

AE and HVR acknowledge support by the Governments of Spain through the Spanish Ministry of Economy and Competitiveness through the project AYA2015-66211-C2-1-P

\section{References}

Abbott B. P., Abbott R., Abbott T. D., Abernathy M. R., Acernese F., Ackley K., Adams C., Adams T., Addesso P., Adhikari R. X., et al. 2016a, ApJ, 818, L22

Abbott B. P., Abbott R., Abbott T. D., Abernathy M. R., Acernese F., Ackley K., Adams C., Adams T., Addesso P., Adhikari R. X., et al. 2016b, Physical Review Letters, 116, 241103

Abbott B. P., Abbott R., Abbott T. D., Acernese F., Ackley K., Adams C., Adams T., Addesso P., Adhikari R. X., Adya V. B., et al. 2017, Physical Review Letters, 118, 221101

Bertin E., Arnouts S., 1996, A\&AS, 117, 393

Cenarro A. J., Moles M., Cristóbal-Hornillos D., Gruel N., Benítez N., Marín-Franch A., 2010, in Modeling, Systems Engineering, and Project Management for Astronomy IV Vol. 7738 of Proc. SPIE, The Javalambre Astrophysical Observatory project. p. $77380 \mathrm{~V}$

de Kool M., 1992, A\&A, 261, 188

Dufour P., Blouin S., Coutu S., Fortin-Archambault M., Thibeault C., Bergeron P., Fontaine G., 2017, in Tremblay P.-E., Gaensicke B., Marsh T., eds, 20th European White Dwarf Workshop Vol. 509 of Astronomical Society of the Pacific Conference Series, The Montreal White Dwarf Database: A Tool for the Community. p. 3

Gentile Fusillo N. P., Gänsicke B. T., Greiss S., 2015, MNRAS, 448, 2260

Gentile Fusillo N. P., Hermes J. J., Gänsicke B. T., 2016, MNRAS, 455, 2295

Girven J., Gänsicke B. T., Steeghs D., Koester D., 2011, MNRAS, 417, 1210

Goliasch J., Nelson L., 2015, ApJ, 809, 80

Green G. M., Schlafly E. F., Finkbeiner D. P., Rix H.-W., Martin N., Burgett W., Draper P. W., Flewelling H., Hodapp K., Kaiser N., Kudritzki R. P., Magnier E., Metcalfe N., Price P., Tonry J., Wainscoat R., 2015, ApJ, 810, 25

Hameury J.-M., Menou K., Dubus G., Lasota J.-P., Hure J.-M., 1998, MNRAS, 298, 1048 
Henden A., Munari U., 2014, Contributions of the Astronomical Observatory Skalnate Pleso, 43, 518

Howell S. B., Nelson L. A., Rappaport S., 2001, The Astrophysical Journal, 550, 897

Kalomeni B., Nelson L., Rappaport S., Molnar M., Quintin J., Yakut K., 2016, ApJ, 833, 83

Kato T., Hambsch F.-J., Dubovsky P. A., Kudzej I., et al., 2015, PASJ, 67, 105

Kato T., Hambsch F.-J., Monard B., Vanmunster T., et al., 2016, PASJ, 68, 65

Knigge C., Baraffe I., Patterson J., 2011, ApJS, 194, 28

Kolb U., 1993, A\&A, 271, 149

Littlefair S. P., Dhillon V. S., Marsh T. R., Gänsicke B. T., Southworth J., Watson C. A., 2006, Science, 314,1578

Maza J., Gonzalez L. E., 1983, IAU Circ., 3854

McAllister M. J., Littlefair S. P., Dhillon V. S., Marsh T. R., Gänsicke B. T., Bochinski J., Bours M. C. P., Breedt E., Hardy L. K., Hermes J. J., Kengkriangkrai S., Kerry P., Parsons S. G., Rattanasoon S., 2017, MNRAS, 467, 1024

Meyer F., Meyer-Hofmeister E., 1984, A\&A, 132, 143

Neustroev V. V., Marsh T. R., Zharikov S. V., et al., 2017, MNRAS, 467, 597

Osaki Y., 1974, PASJ, 26, 429

Pala A. F., Gänsicke B. T., Townsley D., et al., 2017, MNRAS, 466, 2855

Patterson J., 2011, MNRAS, 411, 2695

Patterson J., Thorstensen J. R., Kemp J., 2005, PASP, 117, 427

Rebassa-Mansergas A., Nebot Gómez-Morán A., Schreiber M. R., Gänsicke B. T., Schwope A., Gallardo J., Koester D., 2012, MNRAS, 419, 806

Ritter H., Kolb U., 2003, A\&A, 404, 301

Roeser S., Demleitner M., Schilbach E., 2010, AJ, 139, 2440

Templeton M. R., 2007, AAVSO Alert Notice, 349

The LIGO Scientific Collaboration The Virgo Collaboration 2017, ArXiv e-prints

The LIGO Scientific Collaboration the Virgo Collaboration Abbott B. P., Abbott R., Abbott T. D., Acernese F., Ackley K., Adams C., Adams T., Addesso P., et al. 2017, ArXiv e-prints

Torres G., Andersen J., Giménez A., 2010, A\&A Rev., 18, 67 
Townsley D. M., Gänsicke B. T., 2009, The Astrophysical Journal, 693, 1007

Unda-Sanzana E., Marsh T. R., Gänsicke B. T., Maxted P. F. L., Morales-Rueda L., Dhillon V. S., Thoroughgood T. D., Tremou E., Watson C. A., Hinojosa-Goñi R., 2008, MNRAS, 388, 889

Warner B., 1995, Cambridge Astrophysics Series, 28

Zorotovic M., Schreiber M. R., Parsons S. G., Gänsicke B. T., Hardy A., Agurto-Gangas C., Nebot Gómez-Morán A., Rebassa-Mansergas A., Schwope A. D., 2016, MNRAS, 457, 3867 